\title{
Effects of Fas-Ligand Overexpression on Alveolar Type II Cell Growth Kinetics in Perinatal Murine Lungs
}

\author{
MONIQUE E. DE PAEPE, SHEILA A. HALEY, ZACHARIE LACOURSE, AND QUANFU MAO \\ Department of Pathology [M.E.D.P., Z.L., Q.M.], Women and Infants Hospital, and Departments of Pathology and Laboratory Medicine \\ [M.E.D.P., Q.M.] and Molecular Biology, Cell Biology and Biochemistry [S.A.H.], Alpert Medical School of Brown University, \\ Providence, Rhode Island 02905
}

\begin{abstract}
We determined the time-specific effects of FasL overexpression on perinatal alveolar type II cell growth kinetics. To achieve temporal overexpression of respiratory epithelium-specific FasL expression, tetracycline inducible CCSP-rtTA/FasL-TetOp transgenic mice were given doxycycline (Dox) from gestational d 14 (E14) to E19 (antenatal treatment group), from postnatal d 1 (P1) to P7 (postnatal group), or from E14 to P7 (combined antenatal and postnatal group). Antenatal Dox administration induced an increase of pulmonary FasL mRNA levels in double transgenic animals up to $>300$-fold over single transgenic littermate controls, associated with massive fetal respiratory epithelial apoptosis and excessive postnatal lethality. Although animals from the combined antenatal/postnatal Dox treatment group continued to display evidence of increased apoptosis, there was a paradoxical increase in alveolar type II cell proliferation, resulting in a net increase in type II cell density, elevated pulmonary surfactant protein $\mathrm{C}$ levels and improved postnatal survival. Postnatal Dox administration was also associated with increased type II cell density, although FasL upregulation was more variable. In conclusion, these results, and our previous studies, suggest that FasL signaling has dual timingdependent proapoptotic and proproliferative effects on postcanalicular type II cell kinetics. (Pediatr Res 68: 57-62, 2010)
\end{abstract}

$\mathrm{T}$ he Fas-Fas-ligand (FasL) receptor-mediated death signaling pathway is one of the better characterized apoptotic signaling systems (1). Engagement of the Fas receptor (CD95/ APO1), a member of the tumor necrosis factor receptor superfamily, by its natural ligand, FasL, results in recruitment of two key signaling proteins, the adapter protein FADD (Fasassociated death domain) and the initiator caspase-8, which together form the death-inducing signaling complex (DISC). Proteolytic autoactivation of DISC results in activation of the effector caspases, including the key effector caspase, caspase-3. Activated caspase-3 cleaves DNA repair enzymes, cellular and nuclear structural proteins, endonucleases, and many other cellular constituents, culminating in effective cell death (1).

In addition to its better known apoptotic functions, there is an increasing body of evidence suggesting that Fas/FasL signaling has important nonapoptotic functions as well $(2,3)$. Fas stimulation can enhance proliferation in lymphocytes, fibroblasts, hepatocytes, and cardiomyocytes as well as a wide

Received February 1, 2010; accepted March 17, 2010.

Correspondence: Monique E. De Paepe, M.D., Department of Pathology, Women and Infants Hospital, 101 Dudley Street, Providence, RI 02905; e-mail: mdepaepe@ wihri.org Supported, in part, by NIH P20-RR18728 [M.E.D.P.]. range of tumor cells (4-8). In addition to promoting proliferation and activation, Fas engagement can also trigger inflammatory changes $(9-11)$, induce maturation (12) and stimulate angiogenesis (13).

The Fas/FasL system has been implicated as a critical regulator of alveolar type II cell apoptosis in various clinical and experimental models of adult lung injury (14-18). The role of Fas/FasL signaling in physiologic and pathologic perinatal lung development remains poorly understood. We previously demonstrated that engagement of the Fas receptor in newborn lungs, either by activating anti-Fas antibody (19) or respiratory epithelium-specific FasL overexpression (20), results in increased alveolar epithelial apoptosis and disrupted alveolar remodeling. In more recent studies, we assessed lung growth kinetics in newborn Fas-deficient $l p r$ mice exposed to room air or hyperoxia $\left(95 \% \mathrm{O}_{2}\right)(21)$. In hyperoxic conditions, the rates of lung growth and proliferation were significantly higher in wild-type mice than in $l p r$ mice, suggesting that the predominant function of the Fas/FasL system following hyperoxic injury in newborn lungs is proproliferative, rather than proapoptotic (21). Furthermore, Fas-deficient $l p r$ mice exposed to room air showed transient growth and alveolarization defects during the first month of life, suggesting that in normoxic conditions, as well, Fas/FasL signaling in newborn lungs exerts mainly proproliferative functions, albeit only temporarily (21).

In this study, we used a gain-of-function approach to determine whether the effects of Fas/FasL signaling in perinatal lungs may be age- or developmental stage-specific. To achieve time- and cell-specific FasL-mediated effects, we used a newly generated conditional respiratory epithelium-specific FasL overexpressing strain of transgenic mice (20). In an initial study introducing this novel mouse model, we focused on the apoptotic effects of respiratory epithelium-specific FasL overexpression from gestational d E14 (term $=$ E19) until the end of the first postnatal week (P7) (20). In this study, we extended this preliminary work and compared the effects of antenatal (E14-birth), postnatal (birth-P7), and combined antenatal/ postnatal (E14-P7) FasL overexpression on growth kinetics of lungs and surfactant-producing alveolar type II cells in newborn mice.

Abbreviations: CCSP, Clara cell secretory protein; Dox, doxycycline; rtTA, reverse tetracycline-transactivator; SP-C, surfactant protein-C 


\section{METHODS}

Animal husbandry and tissue processing. We used a tetracyclinedependent tet-on overexpression system to achieve time-specific FasL transgene expression in the respiratory epithelium, as previously described (20). Transgenic (tetOp) ${ }_{7}$-FasL mice (responder line) were crossed with CCSPrtTA mice (activator line) to yield a mixed offspring of double transgenic $\left(\mathrm{CCSP}-\mathrm{rtTA}+/(\text { tetOp })_{7}\right.$-FasL +$)$ and single transgenic (CCSP-rtTA $+/$ $(\text { tetOp })_{7}-\mathrm{FasL}-$ ) littermates. Double transgenic mice will be denoted in the text as "CCSP+/FasL+" mice, whereas single transgenic mice will be denoted as "CCSP+/FasL.- ."

Doxycycline (Dox, $1.0 \mathrm{mg} / \mathrm{mL}$ ) was added to the drinking water of pregnant and/or nursing dams between E14 and E19 (antenatal treatment group), between P1 and P7 (postnatal treatment group), or between E14 and P7 (combined antenatal and postnatal treatment group) (Fig. 1). The progeny (CCSP+/FasL + and CCSP+/FasL-) were killed at E19, P7 [early alveolarization stage (22)], or P21 (advanced alveolarization stage) by pentobarbital overdose. Lungs were processed as described in Ref. 20. All animal experiments were conducted in accordance with institutional guidelines for the care and use of laboratory animals.

Quantitative real-time PCR analysis of FasL gene expression. FasL mRNA levels were quantified by real-time PCR analysis as described in Ref. 20. Relative gene expression ratios were calculated according to the SuperArray-recommended [Delta][Delta] $\mathrm{C}_{\mathrm{t}}$ protocol (23).

Western blot analysis of caspase-3 cleavage. Processing and cleavage of the key Fas-dependent executioner caspase, caspase-3, was assayed by Western blot analysis of lung homogenates as described in Ref. 20. Band intensity was expressed as the combined integrated optical density (IOD) of the $17 \mathrm{kD}$ and $20 \mathrm{kD}$ bands, normalized to the IOD of actin bands (loading control).

Analysis of alveolar type II cell growth kinetics. Alveolar type II cells were identified by immunohistochemical analysis using an antibody against prosurfactant protein-C (pro-SP-C) (Abcam Inc., Cambridge, MA). The number of SP-C-immunoreactive type II cells per $60 \times$ high-power field (type II cell density) was determined in 10 randomly selected microscope fields. To assess alveolar type II cell apoptosis, dUTP-FITC TUNEL was combined with immunohistochemical detection of type II cells as described in Ref. 20. Negative controls included omission of transferase enzyme and/or primary antibody, which abolished all corresponding staining.

To evaluate alveolar type II cell proliferation, we performed sequential double immunofluorescence labeling using the proliferation marker, Ki67 (BD Pharmingen, San Diego, CA) (fluorescein-labeled, green), in combination with anti-pro-SP-C antibody (Cy3-labeled, red). The percentage of Ki67-positive type II cells (type II cell proliferative index) was determined by manual counting in at least 25 randomly selected microscope fields. In addition to type II cell apoptosis and proliferation, the effects of timed FasL

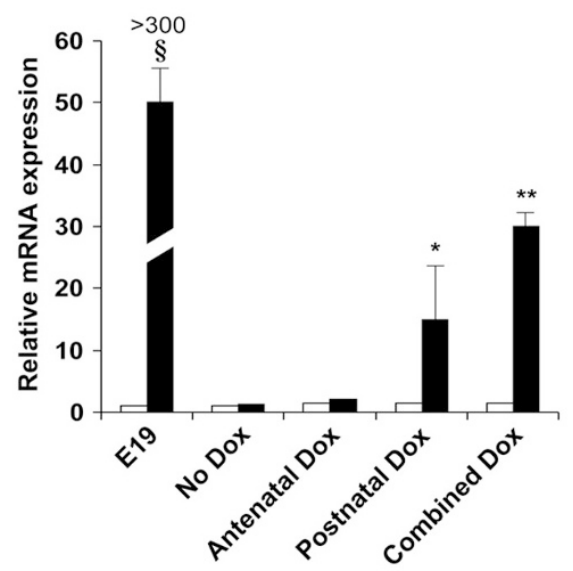

Figure 1. Effect of timed Dox administration on pulmonary FasL mRNA expression. Real-time PCR analysis of FasL mRNA transcripts in lung homogenates of single ( $\square$ ) or double ( $\square$ ) transgenic mice at E19 (first pair) and P7 (remaining pairs). Values represent mean \pm SD of at least five animals per group. At P7, FasL mRNA levels were significantly higher in Dox-treated double transgenic CCSP + /FasL + mice of the postnatal and combined antenatal/postnatal treatment groups compared with their single transgenic littermates. $* p<0.01 ; * * p<0.002 ; \S p<0.0001$ vs corresponding CCSP + / FasL - animals. overexpression on the surfactant system were assessed by Western blot analysis of immunoreactive surfactant protein- $\mathrm{C}$ content in lung homogenates as described in Ref. 21.

Analysis of alveolarization. For morphologic and morphometric studies, lungs were formalin-fixed by standardized tracheal instillation in situ. Alveolarization was quantified by computer-assisted morphometric analysis of the mean cord length (MCL) (20) by a single observer who was unaware of the genotype or experimental condition of the animal analyzed.

Data analysis. Values are expressed as mean \pm SD or, where appropriate, as mean \pm SEM. The significance of differences between groups was determined by ANOVA with post hoc Scheffe test where indicated, and a $p \leq$ 0.05 was considered significant.

\section{RESULTS}

Timed Dox administration provides temporal regulation of pulmonary FasL overexpression in bitransgenic CCSP+/ FasL+ mice. Dox treatment from E14 to E19 induced a robust more than 300-fold increase in pulmonary FasL mRNA levels by $\mathrm{E} 19$ in bitransgenic CCSP $+/ \mathrm{FasL}+$ mice compared with single transgenic CCSP $+/$ FasL - littermates (Fig. 1). Discontinuation of Dox administration at birth in the antenatal Dox treatment group resulted in near-normal pulmonary FasL mRNA levels by P7, whereas continuous Dox administration from E14 to P7 maintained persistently elevated pulmonary FasL mRNA levels as demonstrated at P7. Postnatal Dox administration from $\mathrm{P} 1$ to $\mathrm{P} 7$ resulted in more variable pulmonary FasL mRNA levels, ranging from 5- to 35-fold increases compared with single transgenic littermates (average increase, 15-fold). In the absence of Dox, the FasL mRNA levels of double transgenic littermates were similar to those of single transgenic littermates, indicating that there was no Dox-independent transgene overexpression.

Effect of timed pulmonary FasL overexpression on caspase-3 cleavage. At E19, levels of caspase split products were more than 40 -fold higher in Dox-treated CCSP $+/$ FasL + animals compared with their CCSP $+/$ FasL - littermates (Fig. 2). By P7, the levels of caspase-3 cleavage products in antenatally Dox-treated double transgenic animals had decreased to low levels equivalent to those of single transgenic littermates or non-Dox-treated animals. In contrast, caspase cleavage products remained significantly more than 15 -fold elevated in CCSP+/FasL + animals of the combined antenatal/ postnatal Dox treatment group. In postnatally Dox-treated $\mathrm{CCSP}+/ \mathrm{FasL}+$ animals, caspase cleavage was comparable with that of $\mathrm{CCSP}+/ \mathrm{FasL}-$ littermates.

Effect of timed pulmonary FasL overexpression on lung morphology and alveolar type II cell density. We previously demonstrated that antenatal FasL overexpression induces massive respiratory epithelial apoptosis in double transgenic animals (20). In this study, we assessed the time-specific effects of FasL overexpression on respiratory epithelial apoptosis at P7. Control animals not exposed to Dox and Dox-treated single transgenic animals showed only scattered detached intraalveolar cells (Fig. 3A). In contrast, FasL overexpressing double transgenic animals of all study groups revealed evidence of increased pulmonary apoptosis, although the degree and distribution of apoptotic activity differed among the three treatment groups (Fig. 3B-D). Animals treated with Dox during the antenatal period only showed scattered residual pockets of apoptotic cellular debris, mainly localized to the 


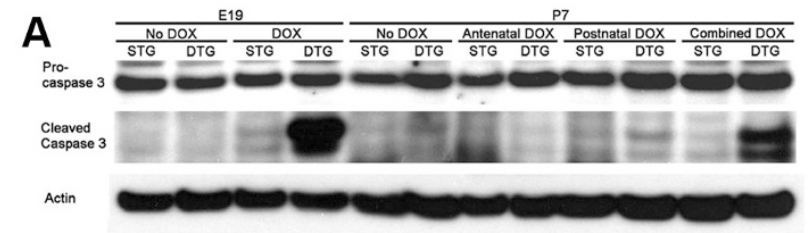

B

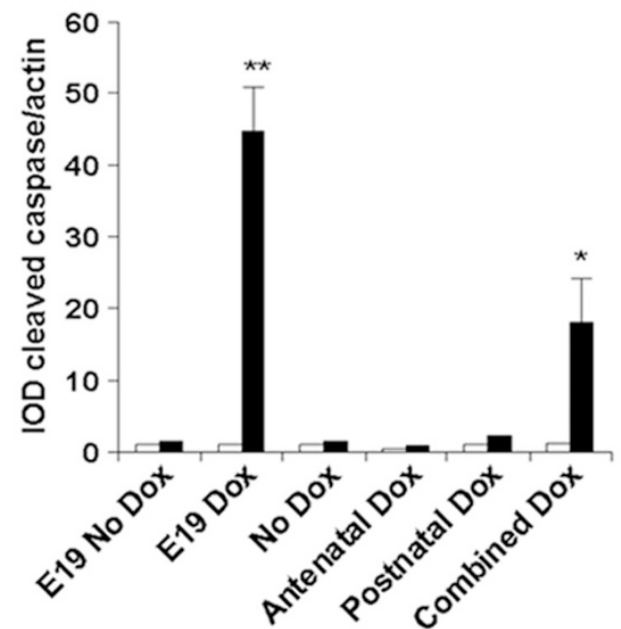

Figure 2. Effect of timed pulmonary FasL overexpression on caspase-3 cleavage. A. Western blot analysis of pulmonary caspase-3 cleavage. STG, single transgenic; DTG, double transgenic. $B$. Densitometry of Western blot. Values represent combined IOD of $17 \mathrm{kD}$ and $20 \mathrm{kD}$ cleavage products expressed as mean \pm SD. At least four single $(\square)$ or double (ם) transgenic animals were studied per group at E19 (first two pairs) or P7 (remaining pairs). Significant caspase cleavage is seen at E19 following antenatal Dox treatment and at $\mathrm{P} 7$ following combined antenatal/postnatal Dox treatment. $* p<0.01 ; * * p<0.001$ vs corresponding CCSP $+/$ FasL - animals.

subpleural airspaces (Fig. 3B). The findings in animals treated with Dox postnatally were more variable and ranged from scattered apoptotic cells to abundant apoptotic cellular debris associated with macrophage collections (Fig. 3C). Lungs of double transgenic mice of the combined antenatal/postnatal treatment group uniformly showed abundant degenerating cells within the airspaces, admixed with macrophages (Fig. 3D).

Alveolar type II cells were identified and quantified by anti-SP-C immunohistochemistry. In non-Dox-treated animals, whether double or single transgenic, alveolar type II cells were regularly distributed along the alveolar walls (Fig. $3 E$ ). In antenatally treated double transgenic animals, the alveolar type II cell density was similar to that of non-Doxtreated animals or Dox-treated single transgenic littermates (Fig. $3 F$ ). The type II cell density was significantly higher in CCSP $+/$ FasL + animals from the postnatal and combined antenatal/postnatal Dox-treatment groups compared with their respective single transgenic littermates (Fig. $3 G$ and $H$ and bottom left bar graph). In these animals, the type II cells were often seen in clusters within the interstitium (Fig. $3 G$ and $H$ ). The type II cells appeared larger and more intensely SP-Cimmunoreactive in double transgenic animals of the combined antenatal/postnatal Dox treatment group compared with animals of other treatment groups (Fig. $3 H$ ).

Effect of timed pulmonary FasL overexpression on alveolar type II cell apoptosis. To determine the apoptotic activity of alveolar type II cells, TUNEL labeling was com-

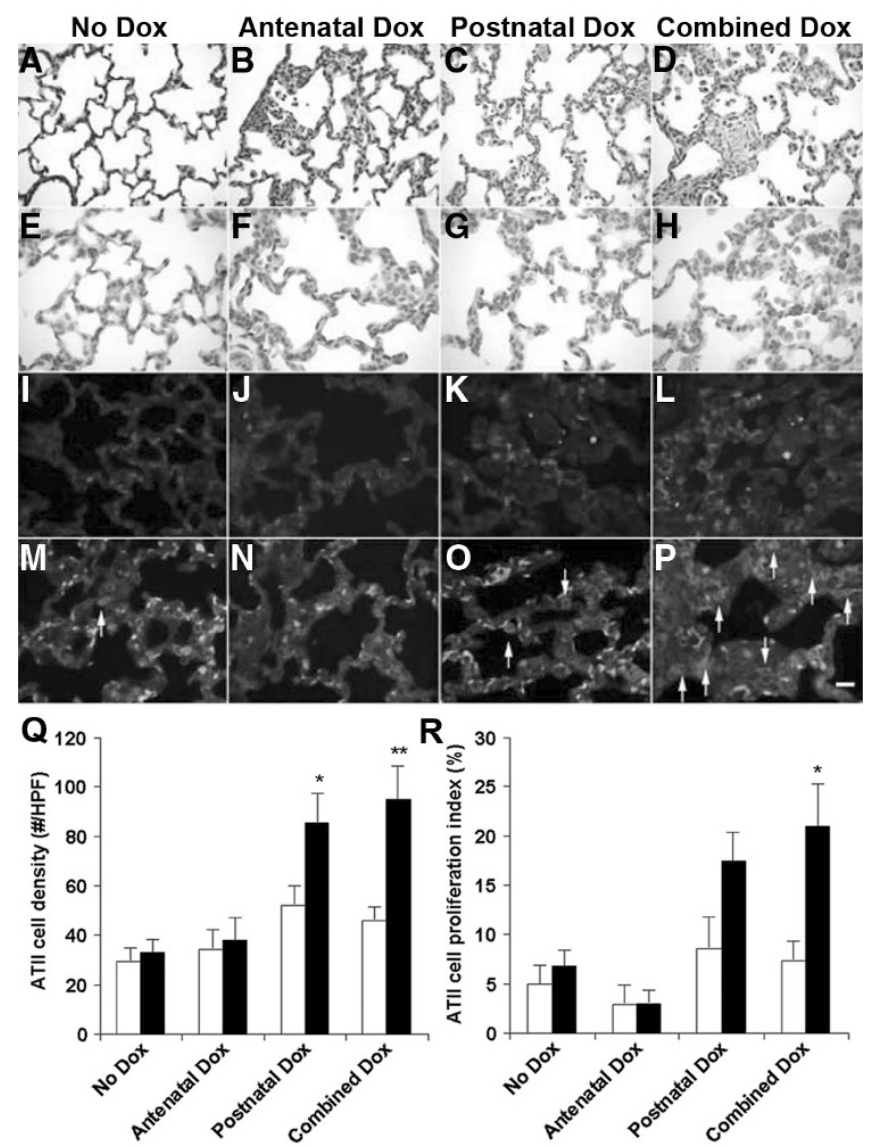

Figure 3. Effects of timed pulmonary FasL overexpression on lung morphology and alveolar type II cell growth kinetics at P7. A-D: Hematoxylin-eosin staining showing unremarkable saccular architecture in the absence of Dox $(A)$ and scattered cellular debris following antenatal $(B)$, postnatal $(C)$ or combined $(D)$ Dox treatment. Original magnification $\times 400$. $E-H$ : Surfactant protein-C immunohistochemistry showing increased numbers of type II cells in animals of the postnatal $(G)$ and combined $(H)$ treatment groups compared with untreated $(E)$ or antenatally treated $(F)$ animals. Avidin-biotinperoxidase/DAB method with hematoxylin counterstain, original magnification $\times 600$. $I-L$ : TUNEL labeling (fluorescein, green) combined with surfactant protein-C immunohistochemistry (Cy3-labeled, red). Increased numbers of apoptotic cells are seen in double transgenic animals of the postnatal $(K)$ and combined $(L)$ treatment groups compared with the untreated $(I)$ and antenatally treated $(J)$ groups. Abundant SP-C-positive and TUNEL-negative type II cells are seen in the postnatal and combined treatment groups. Original magnification $\times 400$. $M-P$ : Ki67 labeling (fluorescein-labeled, green) combined with surfactant protein-C immunohistochemistry (Cy3-labeled, red) showing abundant type II cell proliferation in the combined treatment group $(P)$ compared with untreated $(M)$, antenatally treated $(N)$ or postnatally treated $(O)$ groups. Original magnification $\times 400$. Scale bar $=20 \mu \mathrm{m}$ for all images. $Q$ : Type II cell density (cells per $60 \times$ high-power field) in lungs of single ( $\square$ ) or double (ם) transgenic mice. Values represent mean \pm SD of at least three animals per group. Type II cell density was significantly higher in double transgenic animals of postnatal and combined Dox treatment groups compared with single transgenic littermates. $* p<0.05$; ** $p<0.01$ vs corresponding CCSP+/FasL - animals. HPF: high-power field. $R$. Alveolar type II cell proliferation index (percentage of Ki67-positive type II cells). Values represent mean $\pm \mathrm{SD}$ of at least three animals per group. Type II cell proliferation rates were significantly higher in double transgenic animals ( $\square$ ) of the combined Dox treatment group compared with single transgenic littermates $(\square) . * p<0.05$ vs corresponding CCSP $+/$ FasL - animals.

bined with anti-SP-C immunohistochemistry. We previously demonstrated that Dox administration from E14 to E19 induces massive respiratory epithelial apoptosis and results in a 
dramatic reduction of identifiable alveolar type II cells by E19 (20). The pulmonary apoptotic activity of non-Dox-treated animals and Dox-treated single transgenic animals of any treatment group was low at $\mathrm{P} 7$ and preferentially localized to SP-C-negative stromal cells (Fig. 3I). The apoptotic activity of double transgenic animals of the antenatal treatment group was similar to non-Dox-treated animals, except for the focal presence of apoptotic debris (Fig. 3J). Lungs of double transgenic animals in the postnatal and combined Dox-treatment groups at P7 contained abundant TUNEL-positive cellular debris in the airspaces, although the degree of apoptosis was more variable in the postnatal treatment group (Fig. $3 K$ and $L$ ). At this time point, most intraalveolar apoptotic nuclei were devoid of SP-C immunoreactivity or other identifiable cellular characteristics. Abundant intensely SP-C-immunoreactive type II cells were noted along the alveolar septa and within the alveolar walls in the postnatal and combined antenatal/ postnatal Dox treatment groups. As seen in Fig. $3 K$ and $L$, these hyperplastic type II cells were virtually uniformly TUNEL-negative.

Effect of timed pulmonary FasL overexpression on alveolar type II cell proliferation. The proliferative activity of alveolar type II cells at P7 was assessed by combining antiSP-C labeling with the proliferation marker Ki67. The proliferative activity of postnatal lungs at $\mathrm{P} 7$ was high in all study groups, as expected in actively growing newborn lungs (Fig. $3 M-P)$. In double transgenic pups of the combined Dox treatment group, the proliferative activity of the type II cells was significantly 3 -fold higher than in single transgenic littermates (Fig. $3 P$ and bottom right bar graph). In postnatally treated double transgenic animals, as well, type II cell proliferative activity tended to be higher than in single transgenic littermates (Fig. 3O); however, larger interpup variability precluded statistical significance with the sample sizes studied.

Effect of timed pulmonary FasL overexpression on pulmonary surfactant protein content. To further assess the time-specific effects of FasL overexpression on the surfactant system, we studied pulmonary SP-C protein levels at P7 by Western blot analysis of whole lung homogenates. In the absence of Dox, SP-C-immunoreactive protein levels were similar in double and single transgenic animals (Fig. 4). In litters treated with antenatal Dox only, the pulmonary SP-C protein levels were similar in double and single transgenic animals. In contrast, combined antenatal/postnatal Dox treatment resulted in a dramatic more than 15 -fold increase in pulmonary SP-C protein levels. In double transgenic animals Dox-treated during the postnatal period only, pulmonary SP-C levels tended to be slightly higher than in single transgenic littermates; however, this difference was not significant (Fig. 4).

Effect of timed pulmonary FasL overexpression on neonatal survival. To assess the effects of timed FasL transgene overexpression on postnatal viability, we determined the proportions of double transgenic CCSP + /FasL + and single transgenic CCSP $+/$ FasL - progeny at P21. CCSP-rtTA mice are homozygous and $(\mathrm{TetOp})_{7}$-FasL mice hemizygous for their respective transgenes. Equal proportions of double trans-

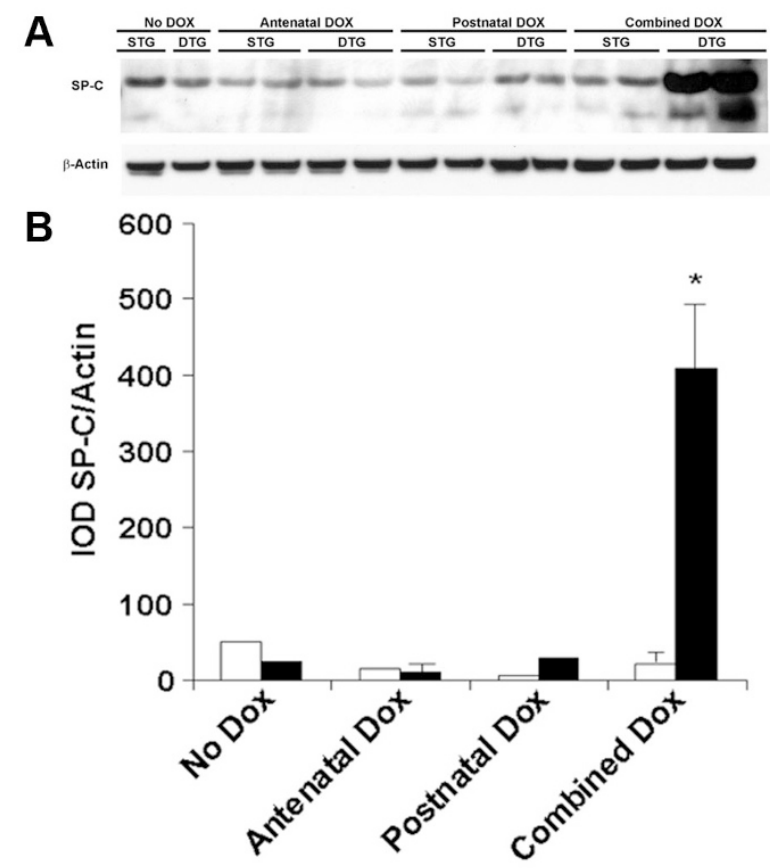

Figure 4. Effect of timed FasL overexpression on pulmonary surfactant protein-C levels. A. Western blot analysis of pulmonary SP-C levels. STG, single transgenic; DTG, double transgenic. $B$. Densitometry of Western blot. Values represent mean \pm SD of at least three single $(\square)$ or double $(\square)$ transgenic animals per group at P7. Pulmonary SP-C levels are significantly higher in double transgenic animals of the combined Dox treatment group compared with single transgenic littermates. $* p<0.01$ vs corresponding $\mathrm{CCSP}+/$ FasL - animals.

Table 1. Proportion of double transgenic CCSP $+/$ Fas $L+$ mice among surviving animals at P21

\begin{tabular}{clc}
\hline Age & \multicolumn{1}{c}{ Dox regimen } & $\begin{array}{c}\text { CCSP }+ \text { FasL }+ \text { fraction } \\
\text { of living offspring }\end{array}$ \\
\hline \multirow{2}{*}{ P21 } & No Dox & $15 / 27(56 \%)$ \\
& Antenatal Dox (E14-E19) & $4 / 25(16 \%)^{*}$ \\
& Postnatal Dox (P1-P7) & $27 / 59(46 \%)$ \\
& Combined Dox (E14-P7) & $8 / 22(36 \%)$ \\
\hline
\end{tabular}

Fraction (percentage) of double transgenic $\mathrm{CCSP}+/ \mathrm{FasL}+$ mice among surviving Dox-treated or non-Dox-treated animals at P21.

$* P<0.01$ vs no Dox (Fisher exact test).

genic $\mathrm{CCSP}+/ \mathrm{FasL}+$ and single transgenic $\mathrm{CCSP}+/ \mathrm{FasL}-$ progeny are therefore expected if the presence and/or expression of the transgene do not carry a lethal effect. At E19 (late gestation), the ratios of double and single transgenic Doxtreated fetuses were about equal, indicating that the CCSP + / FasL + double transgenic status does not confer lethal effects in utero (not shown). By P21, the fraction of double transgenic animals was only $16 \%$ in the antenatal Dox treatment group, indicative of excessive lethality (Table 1). In the combined antenatal/postnatal Dox treatment group, survival tended to be higher than in animals that were Dox-treated in the antenatal period only (fraction of double transgenic animals: 36\%), suggesting continued postnatal FasL overexpression confers partial protection against the adverse effects caused by intrauterine apoptotic injury. The fractions of double transgenic animals in the postnatal Dox treatment group were comparable with those of the non-Dox-treated group. 

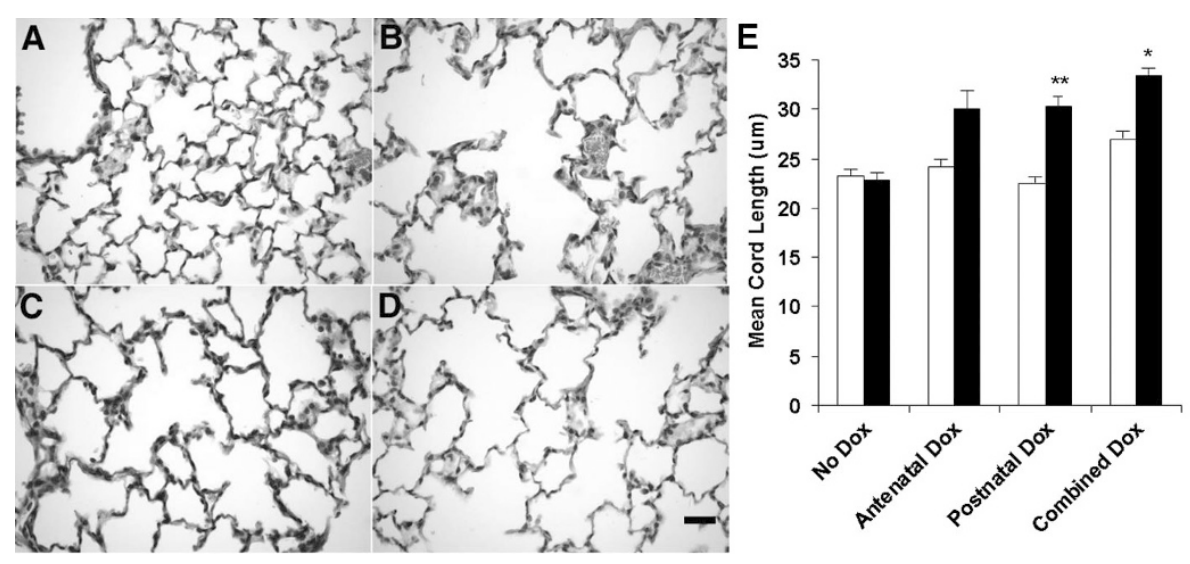

Figure 5. Effect of timed FasL overexpression on lung morphology at P21. Histologic appearance of lungs of double transgenic CCSP $+/$ FasL + mice at P21. Lungs of untreated transgenic mice $(A)$ show well developed alveolar architecture. Lungs of double transgenic mice of the antenatal $(B)$, postnatal $(C)$ or combined $(D)$ Dox treatment groups show markedly enlarged and simplified airspaces. Hematoxylin-eosin staining, original magnification $\times 400$. Scale bar $=20 \mu \mathrm{m}$. E. Mean cord length of lungs of single and double transgenic mice at P21. Values represent mean $\pm \mathrm{SD}$ of at least three single (clear bars) or double (solid bars) transgenic animals per group. ${ }^{*} p<0.01 ; * p<$ 0.001 vs single transgenic animals of same Dox treatment group.
Effect of timed pulmonary FasL overexpression on alveolar remodeling. To determine the effects of timed perinatal FasL expression on alveolar remodeling, lungs of Doxtreated or control transgenic mice were studied at P21. The peripheral lung parenchyma of non-Dox-treated animals or Dox-treated single transgenic mice showed a complex network of small-sized alveoli, separated by delicate septa (Fig. $5 A$ ). Lungs of double transgenic animals of any Dox treatment group displayed marked alveolar disruption characterized by large-sized simplified airspaces and diminished alveolar septation (Fig. $5 B-D$ ). To estimate the degree of alveolarization, the mean cord length (MCL) was determined. The MCL was significantly 25-35\% larger in Dox-treated double transgenic mice of the postnatal and combined Dox treatment groups, confirming the presence of decreased alveolar septation (Fig. 5 , bottom). A similar tendency was noted in the antenatal Dox treatment group (difference not significant).

\section{DISCUSSION}

We determined the effects of temporally regulated FasL overexpression in perinatal lungs on lung growth, surfactant system, and alveolarization. Conditional pulmonary overexpression of FasL was achieved using our newly generated tetracycline inducible respiratory epithelium-specific FasL overexpressing transgenic mice (20). In concordance with our previous study (20), we determined that antenatal FasL overexpression has seemingly exclusive proapoptotic effects, which, as we previously described (20), result in virtual elimination of identifiable alveolar type II cells by the time of birth. Despite postnatal catch-up growth of type II cells to near-normal numbers, antenatal FasL overexpression was associated with a significant increase in postnatal lethality.

Continued pulmonary FasL up-regulation after birth, achieved by uninterrupted Dox exposure from E14 to P7, was associated with persistently elevated levels of respiratory epithelial apoptosis, as demonstrated by the presence of increased caspase cleavage products and increased numbers of TUNEL-positive cells. Despite increased apoptosis, there was a dramatic expansion, rather than further reduction of the alveolar type II cell population. The expansion of the type II cell population-associated with markedly increased pulmonary SP-C levels - was demonstrated to be largely attributable to a robust increase in alveolar type II cell proliferation.
Intriguingly, there was virtually no apoptotic activity in these hyperplastic type II cells despite continuous exposure to high FasL levels. This suggests that the proliferating type II cells emerging upon prolonged FasL exposure may have acquired an altered phenotype resistant to FasL-induced apoptosis.

The intrauterine course of pups from the antenatal Dox treatment group is identical to that of pups from the combined antenatal/postnatal treatment group: in both groups, the lungs are expected to be severely injured at the time of birth due to massive respiratory epithelial apoptosis in utero (20). It is therefore safe to conclude that the observed differences in type II cell population kinetics between these two groups are entirely attributable to distinct postnatal events, namely, markedly different levels of pulmonary FasL expression.

Our study shows that continued postcanalicular FasL overexpression has predominantly proapoptotic effects before birth and switches to predominantly proproliferative effects after birth. The bifunctionality of the Fas/FasL signaling system in this model system is in line with the continuously expanding repertoire of Fas-associated functions. Indeed, although Fas and other death receptors were originally thought to primarily induce apoptotic signaling cascades, recent data indicate that they are capable of initiating multiple signaling pathways. Nonapoptotic functions of the death receptors include regulation of cell proliferation, maturation and differentiation, chemokine production, inflammatory responses, and tumor promoting activities (2-13).

Our findings suggest that the switch of FasL-induced effects on the respiratory epithelium from mainly proapoptotic to mainly proproliferative occurs at the time of the perinatal transition. Although the exact mechanisms remain to be determined, we speculate that increased oxygen tension after birth may play a role in the regulation of the cellular outcome of Fas stimulation. In support of this hypothesis, we recently demonstrated that the Fas/FasL signaling system confers protection against lung injury in hyperoxia-exposed newborn mice, at least in part because of its proproliferative effects in hyperoxic conditions (21). It is not likely that the postnatal type II cell proliferation in the combined treatment group represents a mere rebound phenomenon in reaction to decreasing FasL levels after birth. Indeed, the antenatal treatment group exhibited only modest degrees of reactive type II cell 
proliferation, even though exposed to even more abrupt FasL decreases after birth.

We also determined the effects of exclusively postnatal FasL overexpression by administration of Dox to nursing dams after delivery. Interpretation of the findings in the postnatal Dox treatment group was hampered by limitations inherent to the tetracycline-inducible model system in vivo. The pulmonary FasL up-regulation in postnatally treated animals was less pronounced and showed more interpup variability compared with that achieved by antenatal or combined antenatal/postnatal Dox administration, which we believe to be related to inefficient Dox delivery during lactation compared with transplacental intrauterine transfer to fetal mice. The overall apoptotic and proliferative effects of postnatal Dox administration were less prominent than those seen in the combined antenatal/postnatal treatment group, which may be due to the greater "frontloading" of Dox in the latter group following intrauterine Dox exposure. Nevertheless, the net outcome of postnatal FasL overexpression, similar to animals of the combined treatment group, was increased type II cell density by P7, supporting the notion that postnatal FasL overexpression, however modest, may confer a growth advantage to the type II cell population. In future studies, more consistent Dox administration might be achieved by repeated systemic delivery, although that approach may be technically challenging in newborn mice.

The potential clinical relevance of our findings is important. Antenatal FasL overexpression may demonstrate the pathophysiological consequences of intrauterine conditions that predispose to neonatal lung injury, such as chorioamnionitis or infection. Conversely, postnatal FasL overexpression, corresponding to early saccular stage of development (23- to 29-wk gestation in humans), may replicate the mechanisms of injury following various environmental stressors associated with preterm delivery. The validity of the time-specific FasL overexpressing transgenic mice as model for the various clinical scenarios predisposing to neonatal lung injury is an important experimental and translational goal. However, it is tempting to speculate that in humans, as in mice, the outcome of preterm birth may depend, in part, on the ability of the lung to respond to injury with reactive alveolar type II cell hyperplasia, which, in turn, may depend on developmental stage, presence of antecedent intrauterine injury, and presence and type of postnatal injury.

Interestingly, all Dox treatment regimens resulted in markedly disrupted alveolarization in lungs studied at P21. This strengthens our growing conviction that impairment of alveolar development is the nonspecific final common response of the developing lung to a myriad of insults, mediated by a multitude of disparate mechanisms. We suspect that any disturbance of the delicate balance among cell death, proliferation and differentiation in the epithelial, and presumably also nonepithelial, compartments in postcanalicular lungs may be sufficient to affect alveolar remodeling.
In conclusion, conditional FasL overexpression using a tetracycline-inducible transgenic mouse model has revealed dual proapoptotic and proproliferative functions of the FasL signaling system in perinatal lungs. The molecular regulation and clinical translational implications of the divergent timedependent pulmonary responses to FasL signaling remain to be determined.

Acknowledgments. We thank Dr. J. F. Padbury for review of the article.

\section{REFERENCES}

1. Nagata S 1999 Fas ligand-induced apoptosis. Annu Rev Genet 33:29-55

2. Guicciardi ME, Gores GJ 2009 Life and death by death receptors. FASEB J 23:1625-1637

3. Strasser A, Jost PJ, Nagata S 2009 The many roles of FAS receptor signaling in the immune system. Immunity 30:180-192

4. Alderson MR, Armitage RJ, Maraskovsky E, Tough TW, Roux E, Schooley K, Ramsdell F, Lynch DH 1993 Fas transduces activation signals in normal human T lymphocytes. J Exp Med 178:2231-2235

5. Desbarats J, Newell MK 2000 Fas engagement accelerates liver regeneration after partial hepatectomy. Nat Med 6:920-923

6. Freiberg RA, Spencer DM, Choate KA, Duh HJ, Schreiber SL, Crabtree GR, Khavari PA 1997 Fas signal transduction triggers either proliferation or apoptosis in human fibroblasts. J Invest Dermatol 108:215-219

7. Nelson DP, Setser E, Hall DG, Schwartz SM, Hewitt T, Klevitsky R, Osinska H, Bellgrau D, Duke RC, Robbins J 2000 Proinflammatory consequences of transgenic fas ligand expression in the heart. J Clin Invest 105:1199-1208

8. Shinohara H, Yagita H, Ikawa Y, Oyaizu N 2000 Fas drives cell cycle progression in glioma cells via extracellular signal-regulated kinase activation. Cancer Res 60:1766-1772

9. Choi C, Gillespie GY, Van Wagoner NJ, Benveniste EN 2002 Fas engagement increases expression of interleukin-6 in human glioma cells. J Neurooncol 56:13-19

10. Schaub FJ, Han DK, Liles WC, Adams LD, Coats SA, Ramachandran RK, Seifert RA, Schwartz SM, Bowen-Pope DF 2000 Fas/FADD-mediated activation of a specific program of inflammatory gene expression in vascular smooth muscle cells. Nat Med 6:790-796

11. Jarad G, Wang B, Khan S, DeVore J, Miao H, Wu K, Nishimura SL, Wible BA, Konieczkowski M, Sedor JR, Schelling JR 2002 Fas activation induces renal tubular epithelial cell beta 8 integrin expression and function in the absence of apoptosis. J Biol Chem 277:47826-47833

12. Carlile GW, Smith DH, Wiedmann M 2009 A non-apoptotic role for Fas/FasL in erythropoiesis. FEBS Lett 583:848-854

13. Biancone L, Martino AD, Orlandi V, Conaldi PG, Toniolo A, Camussi G 1997 Development of inflammatory angiogenesis by local stimulation of Fas in vivo. J Exp Med 186:147-152

14. Albertine KH, Soulier MF, Wang Z, Ishizaka A, Hashimoto S, Zimmerman GA, Matthay MA, Ware LB 2002 Fas and fas ligand are up-regulated in pulmonary edema fluid and lung tissue of patients with acute lung injury and the acute respiratory distress syndrome. Am J Pathol 161:1783-1796

15. Hagimoto N, Kuwano K, Nomoto Y, Kunitake R, Hara N 1997 Apoptosis and expression of Fas/Fas ligand mRNA in bleomycin-induced pulmonary fibrosis in mice. Am J Respir Cell Mol Biol 16:91-101

16. Matute-Bello G, Liles WC, Frevert CW, Nakamura M, Ballman K, Vathanaprida C, Kiener PA, Martin TR 2001 Recombinant human Fas ligand induces alveolar epithelial cell apoptosis and lung injury in rabbits. Am J Physiol Lung Cell Mol Physiol 281:L328-L335

17. Matute-Bello G, Liles WC, Steinberg KP, Kiener PA, Mongovin S, Chi EY, Jonas M, Martin TR 1999 Soluble Fas ligand induces epithelial cell apoptosis in humans with acute lung injury (ARDS). J Immunol 163:2217-2225

18. Perl M, Chung CS, Perl U, Lomas-Neira J, de Paepe M, Cioffi WG, Ayala A 2007 Fas-induced pulmonary apoptosis and inflammation during indirect acute lung injury. Am J Respir Crit Care Med 176:591-601

19. De Paepe ME, Mao Q, Embree-Ku M, Rubin LP, Luks FI 2004 Fas/FasL-mediated apoptosis in perinatal murine lungs. Am J Physiol Lung Cell Mol Physiol 287:L730L742

20. De Paepe ME, Gundavarapu S, Tantravahi U, Pepperell JR, Haley SA, Luks FI, Mao Q 2008 Fas-ligand-induced apoptosis of respiratory epithelial cells causes disruption of postcanalicular alveolar development. Am J Pathol 173:42-56

21. Mao Q, Gundavarapu S, Patel C, Tsai A, Luks FI, De Paepe ME 2008 The Fas system confers protection against alveolar disruption in hyperoxia-exposed newborn mice. Am J Respir Cell Mol Biol 39:717-729

22. Amy RW, Bowes D, Burri PH, Haines J, Thurlbeck WM 1977 Postnatal growth of the mouse lung. J Anat 124:131-151

23. Schmittgen TD, Livak KJ 2008 Analyzing real-time PCR data by the comparative C(T) method. Nat Protoc 3:1101-1108 